\title{
Cervical gastrostomy in the surgical treatment of oesophageal cancer by reconstruction with the stomach
}

\author{
JL BARROS
}

From the Department of General Surgery, Hospital Provincial, Ciudad Sanitaria Provincial, Madrid, Spain

\begin{abstract}
A technique of cervical gastrostomy is described. Cervical gastrostomy as an alternative to the use of an indwelling nasogastric tube after cervical oesophagogastrostomy after resection for cancer has been used in six patients and appears to have certain advantages in terms of effective gastric decompression, avoiding discomfort to the patient, and simplification of postoperative nursing. It may play a significant role in reducing postoperative mortality from aspiration pneumonitis and other pulmonary complications.
\end{abstract}

Recent advances in the surgical treatment of oesophageal cancer have led to a higher resectability and reduced postoperative mortality and morbidity rates. Among the most serious and frequent postoperative complications are anastomotic leaks and lung complications. Nowadays, leaks are less frequent as a result of better preoperative preparation, by parenteral feeding, by improved anastomotic techniques and the recent general tendency to perform total oesophagectomy followed by a cervical anastomosis. There are two reasons for this development. First, total oesophageal resection has reduced the incidence of local recurrence of the tumour from submucous longitudinal lymphatic spread. Second, there is the important observation that the mortality rate resulting from a fistula after cervical anastomosis is considerably less than that after an intrathoracic fistula. But postoperative lung complications, chiefly aspiration pneumonitis, also remain a major cause of postoperative difficulties. To a certain extent these can be prevented by better preoperative preparation, intensive pre- and postoperative thoracic physiotherapy and improved anaesthetic techniques, ${ }^{1}$ but in spite of these measures, postoperative lung complications remain a significant cause of death. In a series of cases reported by Belsey, $15 \%$ of the operative fatalities resulted from aspiration pneumonitis (Belsey,

Address for reprint requests: Dr Jose Luis Barros, Cirurgia General, Hospital Provincial, Ciudad Sanitaria Provincial, Calle Dr Esquerdo 46, Madrid-30, Spain. personal communication 1980).

The generally favoured operative technique in the surgical treatment of cancer of the oesophagus today consists of synchronous resection and reconstruction at the same operation, with cervical oesophagogastrostomy or oesophagocolostomy just below the upper oesophageal sphincter. Stomach is the usual substitute for the oesophagus but colon is being used with increasing frequency, the main indication for the use of colon being a history of previous gastric surgery.

Comparing these techniques Skinner ${ }^{2}$ reported three fatalities from aspiration pneumonitis in a series of eight patients on whom a cervical oesophagogastrostomy had been performed by right thoracotomy and a separate cervical incision. He also compared the incidence of aspiration pneumonia after cervical oesophagogastrostomy and after cervical oesophagocolic anastomosis. Out of a total of 18 patients having cervical oesophagogastrostomy, six or $33 \%$ had serious aspiration pneumonia. In a total of 15 patients with cervical oesophagocolic anastomoses, only two had severe aspiration pneumonia. The important role of the upper oesophageal sphincter in protecting the air passages from aspiration and its complications has long been recognised. Interference with the lower oesophageal sphincter by an indwelling nasogastric tube is now recognised as an important cause of severe reflux and oesophageal stricture. In the same way, interference with the function of the upper sphincter by the presence of an indwelling nasogastric tube may 
not only lead to damage to the remaining oesophagus but may also promote a high incidence of pulmonary aspiration. As a result of similar experiences after cervical oesophagogastrostomy and gastric decompression with an indwelling nasogastric catheter, I have devised an alternative technique of cervical gastrostomy avoiding interference with the upper sphincter.

\section{Technique}

After mobilisation of the stomach, by thoracolaparotomy from the left side or by median laparotomy and a separate right thoracotomy, a pyloromyotomy or pyloroplasty is performed. Through a separate cervical incision, on the same side as the thoracotomy, an end-to-side oesophagogastric anastomosis is performed on the posterior aspect of the stomach in one layer. The proximal end of the stomach above the anastomosis is anchored to the prevertebral structures or the anastomosis is covered on its anterior and lateral side with any redundant fundus that is available. Two purse-string sutures are then inserted into the anterior aspect of the stomach and through a small stab incision, a soft rubber catheter is inserted into the stomach, its lower end situated just above the diaphragm or above the pylorus depending on its new situation. The pursestring sutures are tightened onto the drainage tube, care being taken to insure inversion of the gastric mucosa. The gastrostomy thus achieved is anchored to the deeper aspect of the scalene muscles. The end of the tube is then passed through a stab wound in

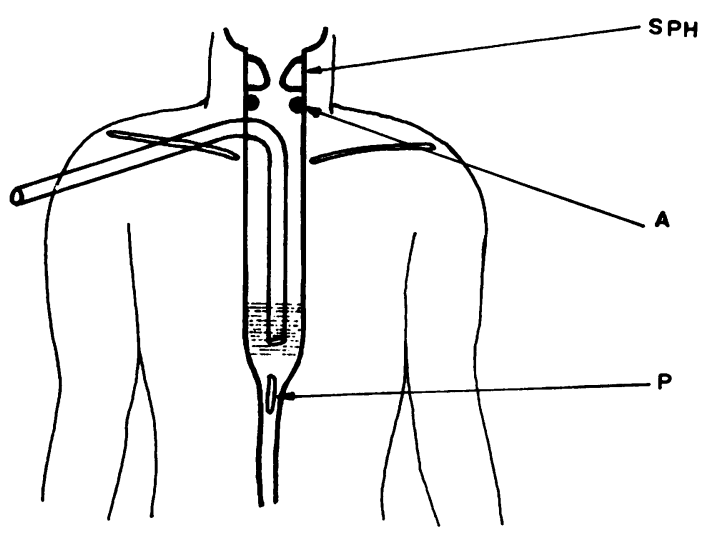

Fig 1 The tube of the cervical gastrostomy is at a distance from the oesophagogastric anastomosis $(A)$ and does not interfere with the sphincter $(S P H)$. The pyloromyotomy is marked $(P)$ and the tube should be near the diaphragm.

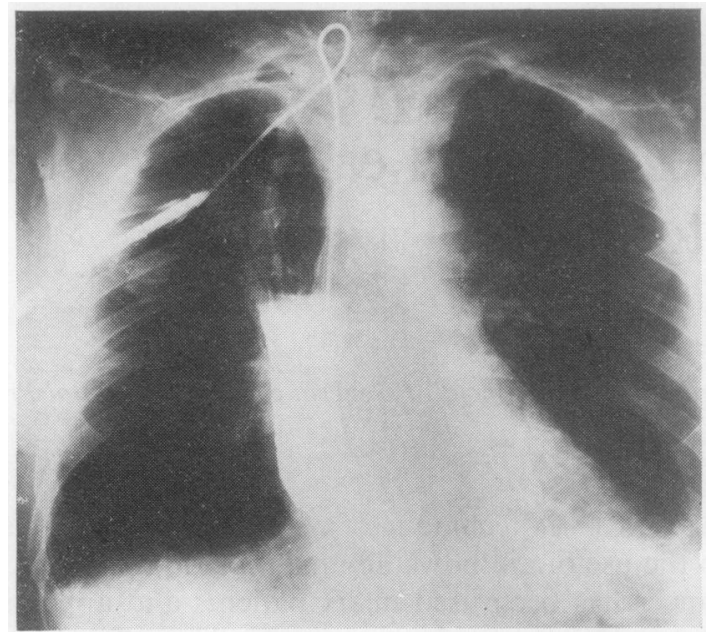

Fig 2 Oral radiological contrast is aspirated through the cervical gastrostomy tube.

the skin of the lower cervical region to which the tube is anchored by a silk stitch (fig 1). The cervical incision is closed. Care is taken to empty the stomach by aspiration before the anaesthetic tube is removed from the trachea. In the postoperative period the gastric contents are drained by aspiration initially and later by gravity (fig 2 ).

The intragastric tube introduced through the cervical gastrostomy is also used for gastric feeding once peristalsis has started, usually on the third postoperative day. If the fluids are well tolerated, on approximately the fourth or fifth day, an indirect laryngoscopy is performed, the vocal cords are examined and if no cord paresis is observed and radiographic studies reveal no anastomotic complications, the patient is allowed to begin taking fluids by mouth.

\section{Patients}

Cervical gastrostomy by this method has been used on six patients after resection of squamous cell carcinoma of the oesophagus. Two were operated upon by the left thoracotomy approach and a separate cervical incision. The other four were treated by median laparotomy, right thoracotomy, and a separate cervical incision. Pyloromyotomy and cervical oesophagogastrostomy were performed on the six patients with the anastomosis on the posterior aspect of the stomach and cervical gastrostomy on the anterior aspect. In all cases, the transplanted stomach was routed via the pleural cavity. 


\section{Results}

The cervical gastrostomy performed in these six cases proved to be an effective method of gastric decompression without any significant complications or discomfort to the patient. No nursing problems were encountered. Five of the cases progressed satisfactorily; one patient died of pulmonary embolism on the eleventh postoperative day. Apart from this patient, no significant lung complications were observed. One patient developed a cervical abscess during the second week but after surgical drainage and removal of the catheter no escape of gastric contents into the neck was observed.

\section{Discussion}

The majority of surgeons, when using the stomach for oesophageal reconstruction, practise some type of gastric drainage procedure. There are however exceptions. ${ }^{3}$ The most common procedures employed are pyloromyotomy or pyloroplasty, with or without the use of an indwelling nasogastric tube. Belsey ${ }^{4}$ uses a pyloromyotomy routinely as this leads to less alkaline reflux than a pyloroplasty. He does not use a nasogastric tube routinely, a tube being inserted only on specific indications. McKeown (personal communication 1980) depending on the type of the pylorus, uses different techniques-a pyloromyotomy for a thickened hypertrophic pylorus, a pyloroplasty in the presence of ulcer scarring, and no drainage procedure when the pylorus appears to be patulous. A nasogastric tube is used in all three instances for the first six postoperative days. Ellis ${ }^{5}$ advocates the use of a nasogastric tube but does not perform any drainage procedure on the pylorus.

Recently, Argov ${ }^{6}$ conducted an important study of 300 patients after upper abdominal surgery. The patients were divided into two equal groups; in one group the nasogastric tube was used routinely and in the second group its use was omitted. The incidence of postoperative pulmonary complications was 10 times higher in those patients treated with an indwelling nasogastric tube than in the group where no tube was used routinely. Belsey also believes that an indwelling nasogastric tube interferes with the competence of the upper oesophageal sphincter and for this reason does not use it routinely after oesophageal reconstruction (personal communication 1980). Paralysis of the vocal cords may develop after operation, either from malignant involvement of the recurrent layrngeal nerve, or from surgical trauma. An indwelling nasogastric tube in this group of patients may lead to an even greater risk of aspiration.

In my experience, the use of cervical jgastrostomy combined with pyloromyotomy or pyloroplasty has certain important advantages over an indwelling nasogastric tube: (1) conservation of the mechanism of the upper oesophageal sphincter; (2) the cervical gastrostomy is situated distal to the upper sphincter and distal also to the oesophagogastric anastomosis thus eliminating possible trauma to the anastomosis by the presence of the tube; (3) the cervical gastrostomy is simple to control and very comfortable for the patient; (4) efficient gastric decompression can be achieved; (5) the removal of the tube is simple, no cervical leak of gastric contents has been observed, and there is no interference with the healing of the cervical incision; (6) the gastrostomy tube remains isolated from the cervical vascular structures and there has been no evidence of trauma to these.

The experience gained with this new technique is so far limited to only six patients, but it appears to be sufficiently advantageous to be considered as an alternative to the use of an indwelling nasogastric tube. It remains to be seen whether its use results in a significant reduction in the still high incidence of aspiration pneumonitis after cervical oesophagogastrostomy.

\section{References}

${ }^{1}$ Palmer KNV, Sellick BA. The prevention of postoperative pulmonary atelectasis. Lancet $1953 ; 1: 164-8$.

2 Skinner DB. Esophageal reconstruction. Am J Surg 1980; 139:789-94.

${ }^{3}$ Angorn IB. Oesophagogastrostomy without a drainage procedure in esophageal carcinoma. Br J Surg 1975;62: 601-4.

4 Belsey RHR. Palliative management of esophageal carcinoma. Am J Surg 1980;139:189-94.

${ }^{5}$ Ellis FH, Gibb P. Esophagogastrectomy for carcinoma. Current hospital mortality and morbidity rate. Ann Surg 1979;190:699-705.

- Argov S, Goldstein I, Barzila O. Is routine use of the nasogastric tube justified in upper abdominal surgery? Am J Surg 1980;139:849-50. 